\title{
İki Ayrık Çift Yıldız XY Ant ve HD 295082'nin İlk Fotometrik
}

\section{Çözümleri}

\author{
Derya SÜRGITT ${ }^{* 1,2}$ \\ ${ }^{1}$ Astrophysics Research Centre and Observatory, Çanakkale Onsekiz Mart University, \\ Terzioğlu Kampüsü, TR-17020, Çanakkale, Turkey \\ 2Department of Space Sciences and Technologies, Faculty of Arts and Sciences, \\ Çanakkale Onsekiz Mart University, Terzioğlu Kampüsü, TR-17020, Çanakkale, Turkey
}

(Alınış / Received: 08.06.2017, Kabul / Accepted: 16.08.2017, Online Yayınlanma / Published Online: 20.09.2017)

Anahtar Kelimeler Özet: Bu çalışmada iki Güney yarımküre çift yıldızının (XY Ant ve Örten Çift Yıldızlar, Temel HD 295082) ilk fotometrik çözümleri sunulmuştur. İki sistemin Parametreler, Yıldızlar, XY Ant ve HD 295082 ASAS $V$ bandı ışık eğrileri Wilson-Devinney kodu ile analiz edilmiştir. XY Ant ve HD 295082'nin fotometrik kütle oranları, sırasıyla, $0.941 \pm 0.019$ ve $1.033 \pm 0.068$ olarak belirlenmiştir. Bu iki ayrık çift sistemin bileşenlerinin mutlak parametreleri tahmin edilmiş ve aynı zamanda evrim durumları tartışılmıștır.

\section{First Photometric Solutions of Two Detached Binaries: XY Ant and HD} 295082

\section{Keywords}

Eclipsing binaries, Fundamental

Parameters,

Stars,

$\mathrm{XY}$ Ant, HD

295082

\begin{abstract}
In this paper, the first photometric solutions of two southern eclipsing binaries (XY Ant and HD 295082) are presented. The ASAS $V$ filter light curves of the two systems were analyzed with Wilson-Devinney code. The photometric mass ratio of XY Ant and HD 295082 were derived to be $0.941 \pm 0.019$ and $1.033 \pm 0.068$, respectively. Absolute parameters of the components of these two detached binaries were estimated and their evolutionary stages are also discussed.
\end{abstract}

*Sorumlu yazar: dsurgit@comu.edu.tr 


\section{Inroduction}

$\mathrm{XY}$ Ant (TYC 7195-2533-1= BV 471= 2MASS J10181223-3648186, $\quad V=9.98$ mag) was first discovered by [21] and classified as a variable star. The system was also listed as a new variable star by [12]. Subsequently, the photographic magnitude, amplitude and period of the system were derived to be $9.5 \mathrm{mag}, 0.7$ mag and 1.837962 days, respectively, by [20]. The period of XY Ant was then corrected to 2.1803 days by [7]. The spectral type of this system is listed as A5 in the SIMBAD data base. Up to now, there have been no published studies on $\mathrm{XY}$ Ant in the literature.

The second target in this paper, HD 295082 (TYC 4789-1175-1=2MASS J06275728-0337316, $V=10.28 \mathrm{mag}$ ), is a fully neglected star. It is only listed as an Algol-type binary star having an orbital period of 2.94304 days in the ASAS (All Sky Automated Survey) data base [15].

The aim of this paper is to determine the absolute parameters of these two neglected southern binary stars and to discuss their evolution stages.

\section{Materials and Method}

\subsection{Materials}

In this study, the ASAS $V$ filter light curves of XY Ant and HD 295082 [15] were used to obtain their photometric solutions. Detailed information about the instrument used and data reduction are given on the web site of ASAS (see http://www.astrouw.edu.pl/ gp/asas/a sas.html).

\subsection{Method}

The ASAS $V$ filter light curves of XY Ant and HD 295082 were analyzed with Wilson-Devinney (W-D) code [25]. In this method, the star surfaces are assumed to be equipotentials. The main light curve fitting parameters are the orbital inclination ( $i)$, surface potential $\left(\Omega_{1,2}\right)$, effective temperature $\left(T_{1,2}\right)$, mass ratio $\left(q=m_{2} / m_{1}\right)$, fractional monochromatic luminosity $\left(L_{1,2}\right), \quad$ limb-darkening coefficient $\left(x_{1}, 2\right), \quad$ gravity-darkening exponents $\left(g_{1,2}\right)$, and bolometric albedos $\left(A_{1,2}\right)$. The subscripts 1 and 2 refer to the primary and secondary components, respectively.

\section{Results}

As a first step, to determine the effective temperature of the primary components, $B$ - $V$ color indices were calculated from the $B$ and $V$ magnitudes of the systems given by [11]. Then, the reduced color excess of $E_{\mathrm{d}}(B-V)$ was derived from the method given by [2]. In this computation, the model reddening of $E_{\infty}(B-V)$ was estimated from maps of infrared dust emission by [19]. Finally, the intrinsic values for the color indices, $(B-V)_{0}$, were computed using the formula of $(B$ $V)_{0}=(B-V)-E_{\mathrm{d}}(B-V)$. Thus, the effective temperatures of the primary components of XY Ant and HD 295082 were estimated to be $6616 \mathrm{~K}$ and $7632 \mathrm{~K}$, respectively, using the computed intrinsic values for the color indices, according to the color index, spectral type and temperature calibrations of [6]. Limb darkening coefficients of the components of the systems were taken from [4] and [5] according to the square-root limb darkening law.

The bolometric gravity-darkening exponents of the components were taken as 0.32 for the convective atmosphere $(T<7200 \mathrm{~K})$ from [13] and as 1.0 for the radiative atmosphere $(T>7200 \mathrm{~K})$ from [24]. The bolometric albedos of the components for XY Ant and HD 295082 were fixed as 0.5 and 1.0 for the convective and radiative atmospheres, 
respectively from [18]. During all iterations, a synchronous rotation and circular orbit for the two systems were assumed.

As a first step, the $q$-search method was carried out to obtain the photometric mass ratios because no known spectroscopic mass ratio exists for the two systems. During the $q$-search procedure, the mass ratio was taken as fixed while the parameters of $i, T_{2}, \Omega_{1}$, $\Omega_{2}$, and $L_{1}$ were adjustable in W-D code. The search was done with detached configuration. As a result, the values of the weighted sum of squares of residuals, $\left(\Sigma W(O-C)^{2}\right)$, between the observed and calculated light curves for each solution versus the related mass ratio $(q)$ values are plotted in Figures 1 and 2 for XY Ant and HD 295082, respectively. As seen from the figures the photometric mass ratios of the systems are around $q=0.94$ and 1.00 for XY Ant and HD 295082, respectively. Therefore, these values for the photometric mass ratios of the systems were taken as initial input parameters in the second (final) step.

In the final step, $i, T 2, q, \Omega_{1}, \Omega_{2}, L_{1}$ and $l_{3}$ were taken as free (adjustable) parameters. However, since the resulting third light contribution was too small (i.e. negligible) and within the standard errors, this was excluded from the list of adjustable parameters. The obtained final results are given in Table 1 . The comparisons of the calculated with the parameters given in Table 1 and observed light curves and the corresponding Roche geometries which obtained with Binary Maker Program (ver. 3.0, [3]) are shown in Figures 3 and 4 for XY Ant and HD 295082, respectively.

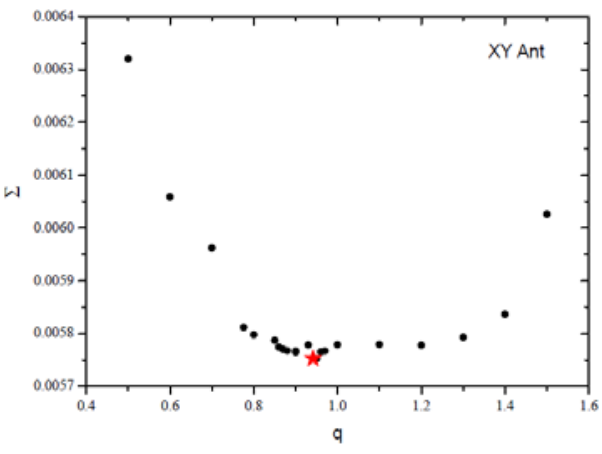

Figure 1. The values of the weighted sum of squares of residuals $\left(\Sigma W(O-C)^{2}\right)$ versus the related mass ratio $(q)$ values in $q$-search procedure for XY Ant

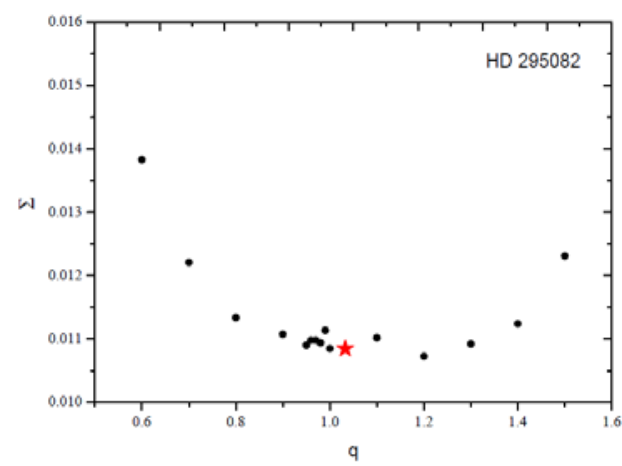

Figure 2. Same as Figure 1 but for HD 295082 
D. Sürgit / First Photometric Solutions of Two Detached Southern Binaries: XY Ant and HD 295082
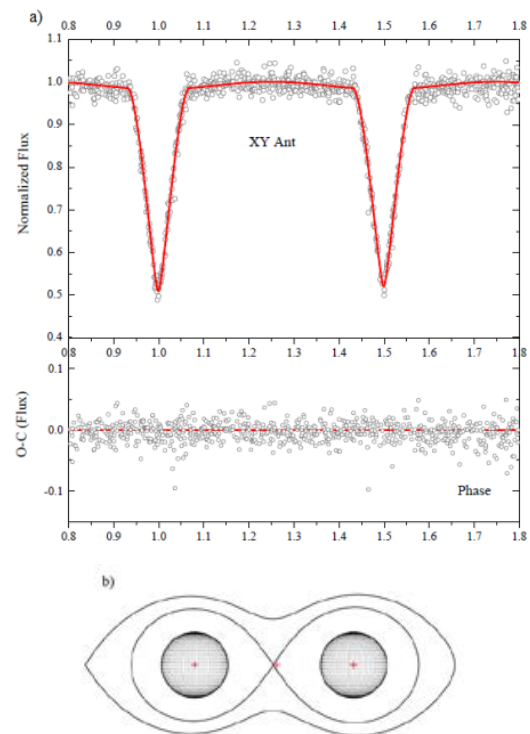

Figure 3. (a) Final model fit to observed light curve, (b) Roche geometry of XY Ant
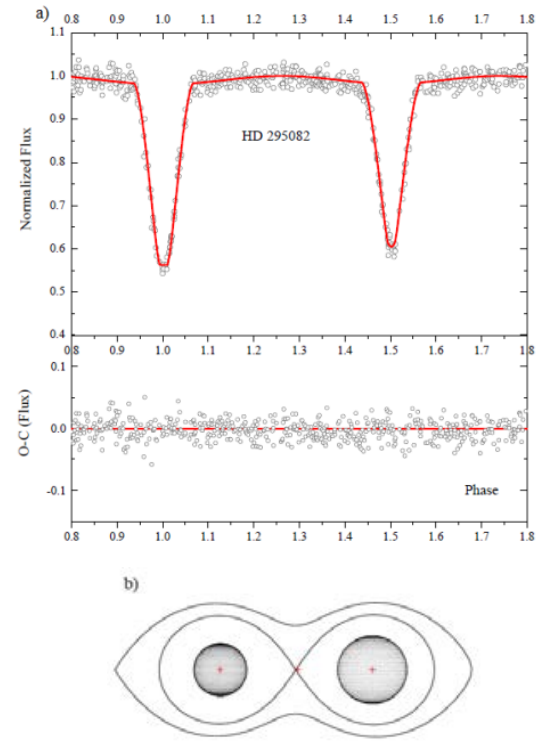

Figure 4. Same as Figure 3 but for HD 295082

Table 1. Parameters of W-D model fit to ASAS V light curves of XY Ant and HD 295082.

\begin{tabular}{ccc}
\hline Parameter & XY Ant & HD 295082 \\
\hline Phase Shift $(\phi)$ & $-0.0010( \pm 0.0001)$ & $0.0020( \pm 0.0002)$ \\
$\boldsymbol{i}\left({ }^{\circ}\right)$ & $88.60( \pm 0.17)$ & $88.98( \pm 0.75)$ \\
$\boldsymbol{T}_{\mathbf{1}}(\mathrm{K})$ & 6616 & 7632 \\
$\boldsymbol{T}_{2}(\mathrm{~K})$ & $6572( \pm 17)$ & $7252( \pm 25)$ \\
$\boldsymbol{q}\left(\boldsymbol{m}_{2} / \boldsymbol{m}_{1}\right)$ & $0.941( \pm 0.019)$ & $1.033( \pm 0.068)$ \\
$\boldsymbol{\Omega}_{1}$ & $5.809( \pm 0.092)$ & $6.849( \pm 0.070)$ \\
$\boldsymbol{\Omega}_{2}$ & $5.613( \pm 0.120)$ & $5.625( \pm 0.250)$ \\
$\boldsymbol{L}_{\mathbf{1}} / \boldsymbol{L}_{\text {tot }}(\boldsymbol{V})$ & $0.501( \pm 0.017)$ & $0.409( \pm 0.005)$ \\
$\boldsymbol{r}_{\mathbf{1}}(\mathbf{m e a n})$ & $0.207( \pm 0.003)$ & $0.173( \pm 0.001)$ \\
$\boldsymbol{r}_{2}(\mathbf{m e a n})$ & $0.207( \pm 0.004)$ & $0.224( \pm 0.021)$ \\
$\boldsymbol{\Sigma} \mathbf{W}(\mathbf{O}-\mathrm{C})^{2}$ & 0.0058 & 0.0108 \\
\hline
\end{tabular}




\section{Discussions and Conclusion}

In this paper, the ASAS $V$ filter light curves of XY Ant and HD 295082 were solved using W-D code. The photometric $q$-search procedure is a generally used method for binary stars which has no spectroscopic observations [e.g., 23, 17]. 2014). Thus, as a first step, since there are no spectroscopic mass ratios and/or published radial velocities for these two systems in the literature, the q-search method was used to obtain their photometric mass ratios. Following this, by including the $q$ parameter in the free/adjustable parameters of iterations, the final photometric solutions of XY Ant and HD 295082 were obtained. As a result, the photometric mass ratios of XY Ant and HD 295082 were obtained to be $0.941 \pm 0.019$ and $1.033 \pm 0.068$, respectively. The final models show that these two systems are detached eclipsing binaries. According to the obtained results, the primary and secondary components of the systems fill their Roche lobes as $64 \%$ and $65 \%$ for XY Ant, and $60 \%$ and $69 \%$ for HD 295082, respectively.

As there are no radial velocity measurements for XY Ant and HD 295082 in the literature, the following method was used to estimate the masses of the components of these neglected systems. The mass of the primary component was estimated from the calibration color index and masses under the assumption that the primary component was a main-sequence star. Then, the mass of the secondary component was computed using the mass ratio and mass of the primary component. Finally, the distance between the centers of the components was calculated from Kepler's third law.

The estimated absolute parameters are given in Table 2 . In the calculations, the solar values of the parameters of effective temperature ( $T_{\text {eff }}$ ), bolometric magnitude $(M \mathrm{bol})$, bolometric correction $(B C)$ and surface gravity $(g)$ were taken from [14]. In order to estimate the uncertainties of the other absolute parameters, a possible error of $0.10 \mathrm{M} \odot$ for the mass values were assumed. Because the modern empirical calibrations of $M$ and $R$ for single mainsequence stars yield $M$ and $R$ within errors of 6 and 3\%, respectively [see, e.g. 22]. On the other hand, considering the error of approximately 0.05 of the color indices given by [11], the error of the effective temperatures of the estimated primary components was taken as $200 \mathrm{~K}$.

To determine the distances of these two systems, the bolometric corrections for the components were derived from the tabulation of [9], according to their effective temperatures. Using the reduced color excess calculated as described in the first paragraph of Section 3, the interstellar extinction was computed with $A_{V}=3.1 \times E(B-V)$ in $V$ band. Then, the distances to XY Ant and HD 295082, which are corrected for interstellar absorption, were predicted as $410 \pm 46$ and $682 \pm 64$ pc using the distance modulus. Also, the eclipseparallax method of [16] gave us $409 \pm 50$ and $686 \pm 66$ pc for the distances to $\mathrm{XY}$ Ant and HD 295082, respectively. On the other hand, according to the GAIA database [10], the distance to the systems are $383 \pm 47$ and $518 \pm 70$ for XY Ant and HD 295082, respectively. 
Finally, there is a very good agreement between distances determining from the photometric parallax method and eclipse parallax method, while there is a discrepancy between distances from GAIA and those based on photometric results for especially HD 295082.

In order to estimate the evolutionary status of these two systems for solar metallicity of $\mathrm{Z}=0.014$ [1], the Geneva evolutionary models [8] were used. For

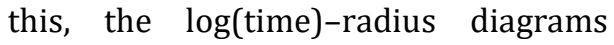
were plotted in Figures 5 and 6 . In these diagrams, the growth of the mean stellar radii for a given stellar mass is displayed (black solid and dashed lines for the primary and secondary component masses, respectively). These diagrams show that the mean dynamic ages are approximately 3.55 and 1.30 Gyr for XY Ant and HD 295082, respectively.
In order to derive more reliable absolute parameters and to discuss their evolution stages, radial velocity measurements for these two neglected systems are necessary.

Table 2. Estimated absolute parameters of XY Ant and HD 295082.

\begin{tabular}{|c|c|c|c|c|}
\hline \multirow[t]{2}{*}{ Parameter } & \multicolumn{2}{|c|}{ XY Ant } & \multicolumn{2}{|c|}{ HD 295082} \\
\hline & $\begin{array}{c}\text { Primary } \\
\text { Component }\end{array}$ & $\begin{array}{l}\text { Secondary } \\
\text { Component }\end{array}$ & $\begin{array}{c}\text { Primary } \\
\text { Component }\end{array}$ & $\begin{array}{l}\text { Secondary } \\
\text { Component }\end{array}$ \\
\hline$A\left(R_{\odot}\right)$ & \multicolumn{2}{|c|}{$9.87( \pm 0.93)$} & \multicolumn{2}{|c|}{$13.29( \pm 2.79)$} \\
\hline$M\left(M_{\odot}\right)$ & $1.4( \pm 0.10)$ & $1.32( \pm 0.12)$ & $1.8( \pm 0.10)$ & $1.85( \pm 0.23)$ \\
\hline$R\left(R_{\odot}\right)$ & $2.07( \pm 0.49)$ & $2.07( \pm 0.59)$ & $2.26( \pm 0.74)$ & $2.92( \pm 1.54)$ \\
\hline $\log g$ (cgs) & $3.95( \pm 0.22)$ & $3.93( \pm 0.21)$ & $3.99( \pm 0.26)$ & $3.77( \pm 0.4)$ \\
\hline$T(\mathrm{~K})$ & $6616( \pm 200)$ & $6572( \pm 201)$ & $7632( \pm 200)$ & $7252( \pm 202)$ \\
\hline$L\left(L_{\odot}\right)$ & $7.42( \pm 1.44)$ & $7.21( \pm 1.98)$ & $15.64( \pm 1.81)$ & $21.19( \pm 2.47)$ \\
\hline$M_{\text {bol }}$ (mag) & $2.58( \pm 0.65)$ & $2.61( \pm 0.75)$ & $1.77( \pm 0.82)$ & $1.44( \pm 1.27)$ \\
\hline$d(\mathrm{pc})$ & \multicolumn{2}{|c|}{$410( \pm 46)$} & \multicolumn{2}{|c|}{$682( \pm 64)$} \\
\hline
\end{tabular}


D. Sürgit / First Photometric Solutions of Two Detached Southern Binaries: XY Ant and HD 295082

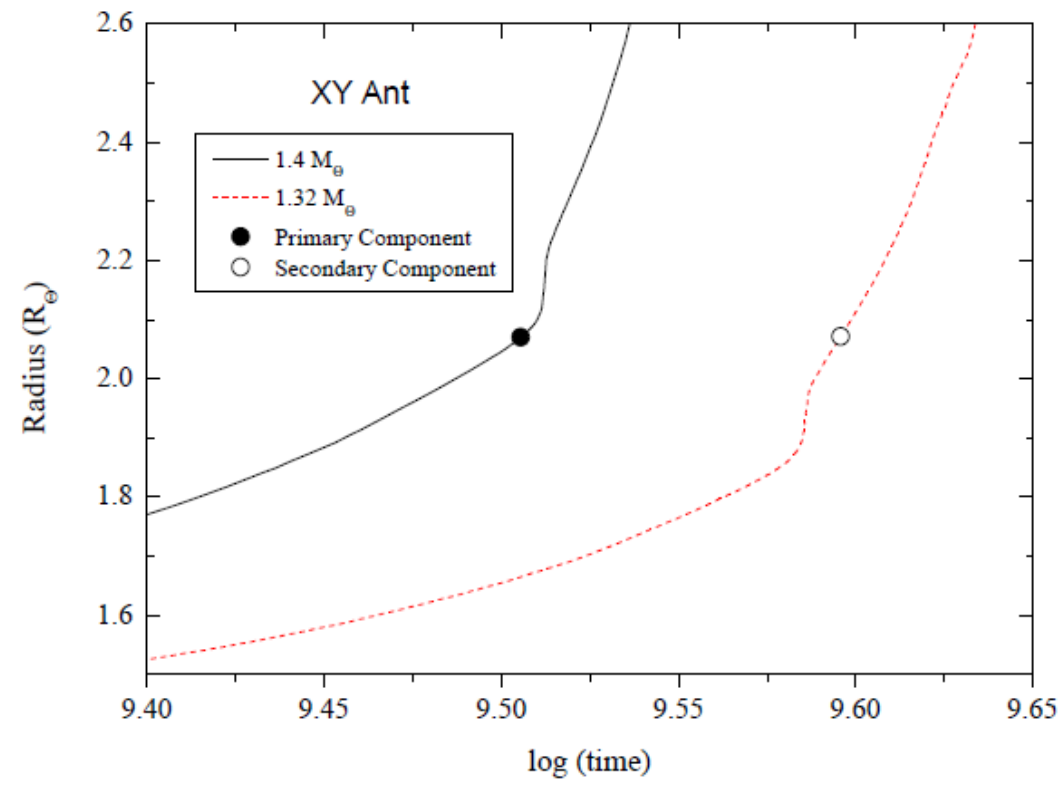

Figure 5. Locations (symbols) of the components of XY Ant in Log(time)-radius diagram and their Geneva evolutionary models (lines)

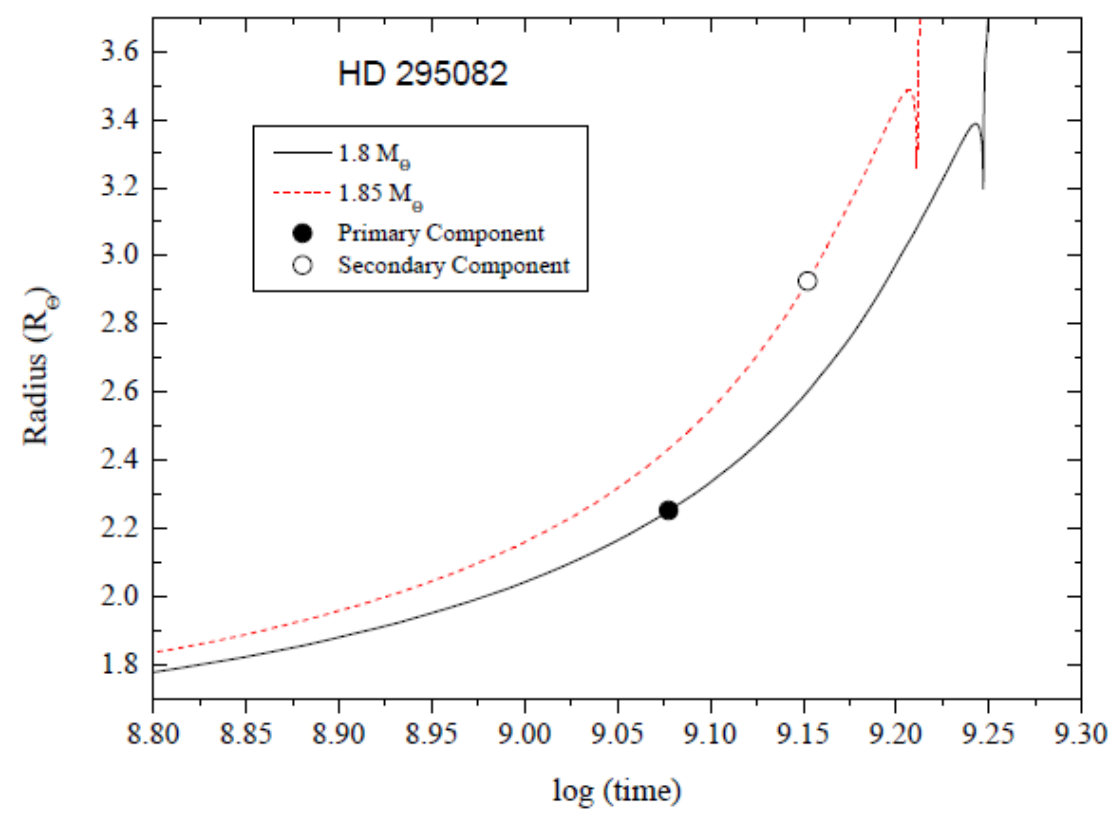

Figure 6. Same as Figure 5 but for HD 295082 


\section{Acknowledgements}

I would like to thank Dr. Ahmet Erdem for his most helpful comments on this study. I also thank Mr. G. H. Lee for checking the English.

\section{References}

[1] Asplund, M. , Grevesse, N. , Sauval, A.J. , Scott, P. , 2009. The Chemical Composition of the Sun. Annual Review of Astronomy and Astrophysics. 47, 481.

[2] Bahcall, J. N., \& Soneira, R. M. 1980. The universe at faint magnitudes. I - Models for the galaxy and the predicted star counts. Astrophysical Journal Supplement Series. 44, 73.

[3] Bradstreet, D. H. and Steelman, D. P. 2002. Binary Maker 3.0 - An Interactive Graphics-Based Light Curve Synthesis Program Written in Java. American Astronomical Society, 201st AAS Meeting, id.75.02; Bulletin of the American Astronomical Society. 201, 7502.

[4] Claret, A. and Bloemen, S. 2011. Gravity and limb-darkening coefficients for the Kepler, CoRoT, Spitzer, uvby, UBVRIJHK, and Sloan photometric systems. Astronomy and Astrophysics. 529, 75.

[5] Claret, A., Hauschildt, P. H., Witte, S. 2013. New limb-darkening coefficients for Phoenix/1d model atmospheres. II. Calculations for $5000 \mathrm{~K} \leq \mathrm{T}_{\text {eff }} \leq 10000 \mathrm{~K}$ Kepler, CoRot, Spitzer, uvby, UBVRIJHK, Sloan, and 2MASS photometric systems. Astronomy and Astrophysics. 552, 16.

[6] Drilling, J.S. , Landolt, A.U. , 2000. In: Arthur N., Cox (Ed.), Allen's Astrophysical Quan- tities, fourth. AIP Press, Springer, New York ISBN: 0-387-98746-0 .

[7] Dvorak, S. W. 2004. Updated Elements for Southern Eclipsing
Binaries. Information Bulletin on Variable Stars. 5542, 1.

[8] Ekström, S. et al. 2012. Grids of stellar models with rotation. I. Models from 0.8 to $120 \mathrm{M} \odot$ at solar metallicity $(Z=0.014)$. Astronomy and Astrophysics. 537, 146.

[9] Flower, P. J. 1996. Transformations from Theoretical HertzsprungRussell Diagrams to ColorMagnitude Diagrams: Effective Temperatures, B-V Colors, and Bolometric Corrections. Astrophysical Journal. 469, 355.

[10] Gaia, C., et al. 2016. Gaia Data Release 1. Summary of the astrometric, photometric, and survey properties. Astronomy and Astrophysics. 595A, 1-1.

[11] Hog, E., et al. 2000. The Tycho-2 Catalogue of the 2.5 million brightest stars. Astronomy and Astrophysics. 355, L27.

[12] Kukarkin, B. V. et al. 1968. Identification List of the New Variable Stars Nominated in 1968. Information Bulletin on Variable Stars. 311, 1.

[13] Lucy, L. B. 1967. Gravity-Darkening for Stars with Convective Envelopes. Zeitschrift für Astrophysik. 65, 89.

[14] Pecaut, M. J., and Mamajek, E. E. 2013. Intrinsic Colors, Temperatures, and Bolometric Corrections of Pre-main-sequence Stars. The Astrophysical Journal Supplement. 208, 9.

[15] Pojmanski, G. 2002. The All Sky Automated Survey. Catalog of Variable Stars. I. $0^{\mathrm{h}}-6^{\mathrm{h}}$ Quarter of the Southern Hemisphere. Acta Astronomica. 52, 397.

[16] Popper, D. M. 1998. HIPPARCOS Parallaxes of Eclipsing Binaries and the Radiative Flux Scale. The Publications of the Astronomical Society of the Pacific. 110, 919.

[17] Prasad, V. et al. 2014. Photometric and polarimetric studies of three $\mathrm{W}$ 
UMa-type binaries: FZ Ori, V407 Peg and LP UMa. Astrophysics and Space Science. 353, 575-594.

[18] Rucinski, S. M. 1969. The Proximity Effects in Close Binary Systems. II. The Bolometric Reflection Effect for Stars with Deep Convective Envelopes. Acta Astronomica. 19, 245.

[19] Schlegel, D.J., Finkbeiner, D.P., Davis, M., 1998. The Astrophysical Journal. Maps of Dust Infrared Emission for Use in Estimation of Reddening and Cosmic Microwave Background Radiation Foregrounds. 500, 525.

[20] Strohmeier, W. and Knigge, R. 1969. Results of the Bamberg Southern Hemisphere Sky Patrol [erratum: 1969MNSSA..28...79S]. Monthly Notes of the Astron. Soc. Southern Africa. 28, 75.

[21] Strohmeier, W., Knigge, R., Ott, H. 1964. Bright Southern BV-Stars. Information Bulletin on Variable Stars. 66, 1.

[22] Torres, G., Andersen, J., Giménez, A. 2010. Accurate masses and radii of normal stars: modern results and applications. The Astronomy and Astrophysics Review. 18, 67-126.

[23] Zola, S., Kolonko, M., Szczech, M. 1997. Analysis of a photoelectric light curve of the W UMa-type binary ST Ind. Astronomy and Astrophysics. 324, 1010-1012.

[24] von Zeipel, H. 1924. The radiative equilibrium of a rotating system of gaseous masses. Monthly Notices of the Royal Astronomical Society. 84, 665.

[25] Wilson, R. E. and Devinney, E. J. 1971. Realization of Accurate Close-Binary Light Curves: Application to MR Cygni. Astrophysical Journal. 166, 605. 